\title{
The Atmospheric Scanning Electron Microscope (ASEM) observes the axonal compartmentalization and microtubule formation in neurons.
}

\author{
Takaaki Kinoshita ${ }^{1}$, Chikara Sato $^{2}$, and Shoko Nishihara ${ }^{1}$ \\ 1. Laboratory of Cell Biology, Department of Bioinformatics, Graduate School of Engineering, Soka \\ University, 1-236 Tangi-machi, Hachioji, Tokyo 192-8577, Japan. \\ 2. Biomedical Research Institute, National Institute of Industrial Science and Technology (AIST), \\ Tsukuba, 305-8566, Japan.
}

The Atmospheric Scanning Electron Microscope (ASEM) is a new Correlative Light-Electron Microscope (CLEM) [1]. Cells can be cultured and differentiated directly on the detachable ASEM dish in a $\mathrm{CO}_{2}$ atmosphere if required. After fixation, cells immersed in radical scavenger solution can be observed in situ by optical microscope (OM) and SEM in a completely correlative manner. As ASEM dish can be coated with various coating reagents, the range of cultivable cell types has been increased, including primary neurons, which allow for the analyses of axonal segmentation and microtubule formation.

In Drosophila and mammalian neurons, some membrane molecules including axon guidance receptors are localized within proximal or distal region of axons autonomously [2, 3]. Drosophila primary neurons obtained from embryos were cultured on a poly-DL-ornithine-coated ASEM dish. After fixation, T antigen and HRP antigen were immunolabeled in the neurons. OM showed that $\mathrm{T}$ antigen, mucin-type $O$-glycan, was specifically localized within the proximal axon segment, whereas HRP antigen, a Drosophila neuronal marker and $\mathrm{N}$-glycan, was localized in the whole axon (Fig. 1A). ASEM revealed a circular pattern localization of $\mathrm{T}$ antigens at the intra-axonal boundary (Fig. 1B; open arrowhead). After observation using ASEM, the neurons were counter-stained by the modified NCMIR method (Fig. 1C). Tubulin was immnolabeled in a neuron with axonal segmentation. Two tubulin bundles were observed in the axon (Fig. 1E; filled arrowhead) and they seemed to be in contact with each other at the boundary (Fig. 1E; open arrowhead) defined by $\mathrm{T}$ antigen localization (Fig. 1D; open arrowhead). These characteristic structural features near the boundary might reflect the mechanism of proximal localization of $\mathrm{T}$ antigen.

Spectraplakin is called as Short stop (Shot) in Drosophila. Shot is a cross-linker protein between F-actin and tubulin and has several functions in neurons [4]. Recently we have reported that Shot mediates proximal localization of $\mathrm{T}$ antigens [5]. In this study, we clarified that Shot is involved in microtubule bundle formation, by using two types of shot null mutants, shot $t^{3}$ and $s h o t^{S F 20}$. The neurons derived from the shot mutants Drosophila were immunostained by anti-tubulin or anti-HRP antigen antibody (Fig. 2A,D). ASEM showed the following phenotypes of abnormal microtubule formation in shot mutant neurons: in shot $^{3}$ mutant, spread-out morphology (Fig. 2B; arrow), disconnection (Fig. 2C; arrow), and several gaps (Fig. 2C; filled arrowheads). shot ${ }^{S F 20}$ mutant has a loop formation (Fig. 2E; arrowhead) and making distance between each microtubule (Fig. 2F; arrow). A spread-out morphology of microtubule was observed in more than $85 \%$ of mutant axons (Fig. 2G). Moreover, the abnormal microtubule formation was rescued by the expression of wild type Shot in shot mutant background (Fig. 2G; Rescue). Therefore ASEM allowed us to clarify that Shot is required for the healthy microtubule bundle formations in neurons. 


\section{References}

[1] H. Nishiyama et al., J Struct Biol 169, (2010) 438-449.

[2] J. Dadd et al., Neuron 1, (1988) 105-116.

[3] T. Katsuki et al., Neuron 64, (2009) 188-199.

[4] N. Sanchez-Soriano et al., J Cell Sci 122, (2009) 2534-2542.

[5] T. Kinoshita et al., Sci Reports 7, (2017) 41455.
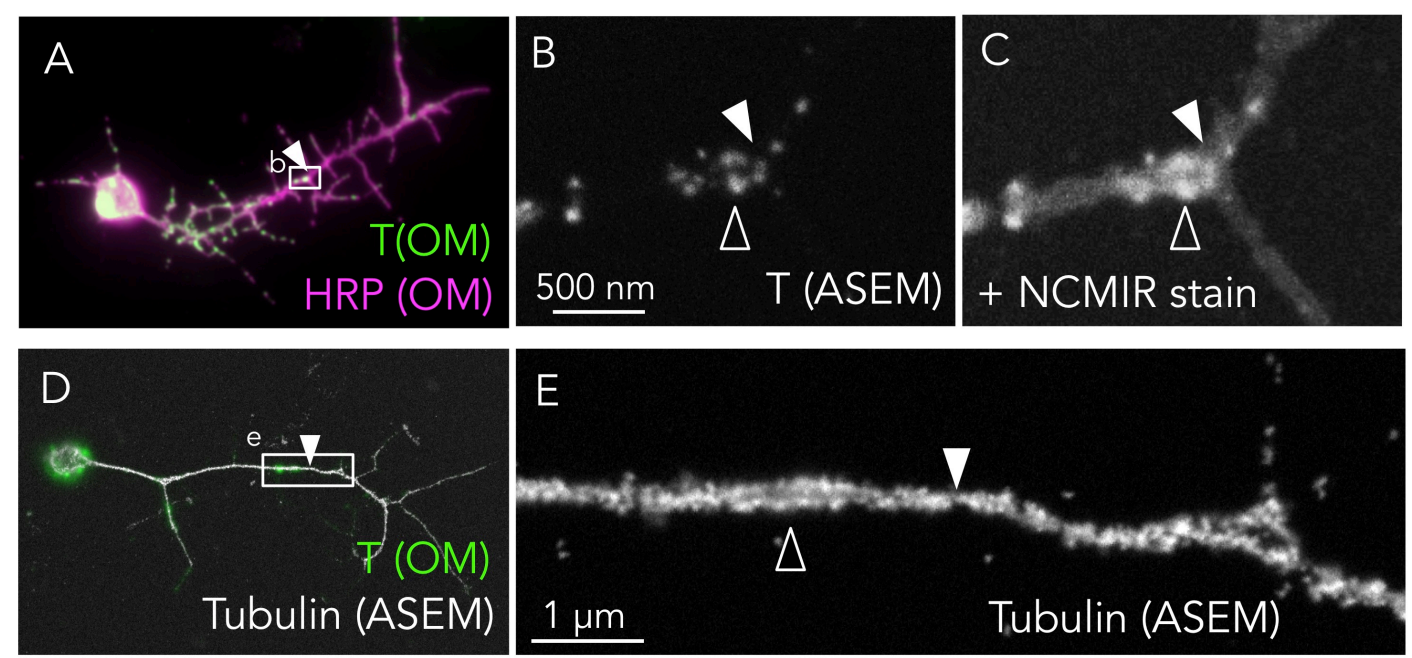

Figure 1. Axonal segmentation in wild-type primary cultured neurons. (A) Localization of T (green) and HRP (magenta) antigens. Filled arrowheads indicate the intra-axonal boundary. (B) High-magnification ASEM image of rectangle $b$ in $A$. The open arrowhead indicates the characteristic structure of $T$ antigens. (C) Field in B after counter-staining by the modified NCMIR method for visualizing the cytoplasm. (D) Overlay image of OM ( $\mathrm{T}$ antigen) and ASEM (tubulin) in a neuron. (E) Highmagnification ASEM image of the rectangle e in D. The two tubulin bundles (open arrowhead) in the axon are in contact at the intra-axonal boundary (filled arrowhead).
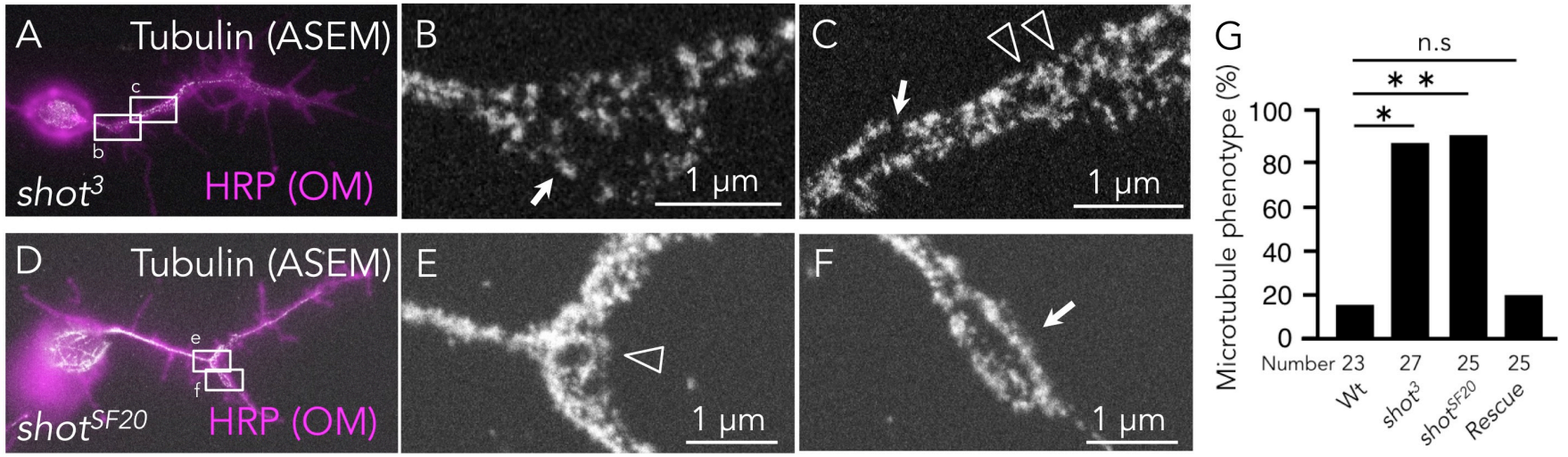

Figure 2. Microtubule formation in neurons derived from shot mutants Drosophila. (A-F) ASEM images showing the localization of $\alpha$-tubulin in cultured neurons of the $\operatorname{shot}^{3}$ (A-C) and $s h o t^{S F 20}$ (D-F) mutants. Arrows and arrowheads reveal defects in microtubule bundles. (A, D) OM image of the $\alpha-$ tubulin (magenta) was merged with HRP antigen distributions imaged by ASEM (white). (B, C) High magnification of rectangles $b$ and $c$ in A. (E, F) High magnification ASEM image of the rectangle e and $\mathrm{f}$ in D. (G) Histogram showing the percentage of abnormalities in microtubule formation. $P$-values were calculated using the G-test. ${ }^{*} p=3.7 \times 10^{-8}, * p=8.1 \times 10^{-8}$. 